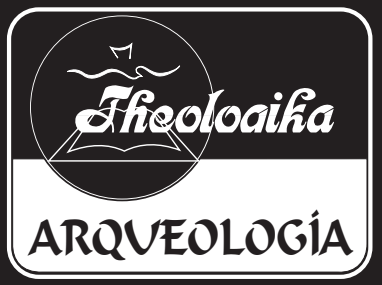

\title{
Laquish en la Edad de Hierro: Identificación del Laquish bíblico
}




\section{Teófilo Correa}

Universidad Peruana Unión 


\section{RESUMEN}

“Laquish en la Edad de Hierro: Identificación del Laquish bíblico" - Algunos eruditos han puesto en tela de juicio el relato del exilio babilónico y la existencia del pueblo Judío en esa época; relegando esto a una historia ideológica del período helenístico. Este trabajo intenta hacer una recapitulación del trabajo arqueológico realizado en Laquish, poniendo especial atención a la edad de hierro especialmente al período B y C. Estimando a la postre la relevancia del sitio, como eje evidentemente histórico del Judá del siglo X al VII. Este autor espera que el mensaje de las piedras pueda ser tenido en cuenta por aquellos que hoy empiezan a ser seducidos por un relato reduccionista del registro bíblico.

\section{SUMMARY}

"Lachish in the Iron Age: Identification of the Biblical Lachish" - Some scholars have questioned the story of the Babilonian exile and even the existence of the Jewish people in that age, reducing all of this to an ideological history in the Hellenistic period. This study will revisit the archaeological work made at Lachish, emphasizing the Iron Age, specifically the period B and C. And will consider, at its end, the relevance of the site. The author has the intention of making the message of the stones more relevant to those who have begun to be seduced by a reductionist story of this Biblical record. 


\section{LAQUISH EN LA EDAD DE HIERRO: IDENTIFICACIÓN DE LAQUISH BÍBLICO}

El nombre Laquish (como aparece en las versiones en español) aparece 22 veces mencionado en las Sagradas Escrituras. ${ }^{1}$ Ésta era una ciudad fortificada en Judá ubicada a unos $55.5 \mathrm{~km}$. al suroeste de Jerusalén y 35 $\mathrm{km}$. desde la costa del Mediterráneo ${ }^{2}$ aproximadamente. Con relación al tiempo que nos ocupa, Hierro II, Laquish constituía la segunda ciudad en importancia después de Jerusalén.

La ciudad ha sido identificada con el Tel Laquish o Tell ed-Duweir en arábigo, llamado ed-Duweir por una villa cercana que lleva este nombre, ${ }^{3}$ está ubicada en el borde de Nahal Laquish (Wadi Ghafr), a través de la cual, pasaba una ruta desde la llanura costanera hasta las montañas de Hebron. ${ }^{4}$

${ }^{1}$ En Josue 10:3, 5, 23, 31, 32, 33, 34, 35; 12:11; 15:39; 2 Rey 14:19; 18:14,17; 19:8; 2 Cró 11:9; 25:27; 32:9; Neh 11:30; Isa 36:2; 37:8; Jer 34:7; Miq 4:13.

${ }^{2}$ Harris Stephen L., Understanding the Bible (Palo Alto, CA: Mayfield Publishing Company, 1985), 399.

${ }^{3}$ Anson F. Rainey, "Historical Geography," en Joel F. Drinkard, Jr., et al., eds., Benchmarks in Time and Culture (Atlanta, Georgia: Scholars Press, 1988), 362.

"David Ushishkin, "Lachish," en Ephraim Stern, ed.; The New Encyclopedia of Archaeological Excavations in the Holy Land, 3:897-911 (Jerusalem: The Israel Exploration Society, 1993), 897. En adelante NEAEHL. 
Fue en 1929 que W. F. Albright sugirió Tel edDuweir como el sitio de Laquish, basando su identificación en Eusebio, quien declaró que Laquish era una villa en la séptima milla desde Eleutheropolis (Beth Guvrin) a Gaza. Aunque la sugerencia estaba basada en evidencias circunstanciales solamente, ${ }^{5}$ fue confirmada por hallazgos arqueológicos tal como una inscripción descubierta in situ ${ }^{6}$ que se verá más adelante. Davies señala que a la luz de las excavaciones británicas apoyadas por la universidad de Tel Aviv no hay duda de ello. ${ }^{7}$ Hoy la mayoría de eruditos acepta la identificación como acertada.

\section{Expediciones}

Laquish fue por primera vez excavada en 1932 por una expedición británica encabezada por James L. Starkey $^{8}$ y auspiciada por un grupo de benefactores privados, incluyendo a Sir Charles Marston. ${ }^{9}$ Starkey dirigió 6 temporadas de excavaciones en Tel ed-Duweir desde $1932^{10}$ hasta que él fue asesinado en una brutal

"Ushishkin, "Lachish" NEAEHL, 897.

${ }^{6}$ Rainey, "Historical Geography", 362.

${ }^{7}$ Graham I. Davies, "British Archaeologists," en Joel F. Drinkard Jr., et al., Benchmarks in Time and Culture (Atlanta, Georgia: Scholars Press, 1988), 44.

${ }^{8}$ Ushishkin, "Lachish" NEAEHL, 897.

${ }^{9}$ Davies, "British Archaeologists", 45.

10Philip J. King, "American Archaeologists," en Joel F. Drikard Jr., et al., eds., Benchmarks in Time and Culture (Atlanta, Georgia: Scholars Press, 1988), 22. 
emboscada en su camino a Jerusalem en $1938 ;{ }^{11}$ siendo supervisado el trabajo final por C. H. Ingey G. L. Harding quienes con Olga Tufnell tomaron la responsabilidad de la publicación de los resultados.

Según informa Davies en su ensayo acerca de las expediciones británicas, Starkey comenzó su trabajo en las áreas alrededor del montículo. ${ }^{12}$ En la primera temporada, él había excavado dos secciones que dieron una clara indicación de la historia del montículo, concentrando su atención posteriormente en la defensa, principalmente en las murallas y en las puertas de la edad de Hierro. ${ }^{13}$

Calificando el trabajo de Starkey, Ushishkin precisa que para su tiempo Laquish fue una excelente excavación y las conclusiones estratigráficas de Starkey han mantenido trascendencia para las futuras excavaciones. ${ }^{14}$

Una excavación de menor rango patrocinada por la Universidad Hebrea y la Universidad de Tel Aviv fue llevada a cabo por Yohani Aharoni en el área del altar sagrado en 1966 y 1968. Ahoroni quien también excavaba en Arad sugirió que el templo encontrado allí datado de ese mismo período del reino de Judá, era similar al santuario de Laquish. Aharoni con su equipo suministraron varios hallazgos desde el nivel VI-I, incluyendo un cuarto de culto en el nivel V. ${ }^{15}$

${ }^{11}$ Davies, "British Archaeologists", 45.

${ }^{12} I b i ́ d$. Lo mismo precisa Ushishkin, en "Lachish", NEAEHL, 897.

${ }^{13}$ Davies, "British Archaeologists", 45.

${ }^{14}$ Ushishkin, "Lachish", NEAEHL, 897.

${ }^{15}$ Ibíd, 898. 
El interés por Laquish fue renovado por una excavación comenzada en 1974 bajo la dirección de David Ushishkin de la Universidad de Tel Aviv, ${ }^{16}$ asistido por G. Barkay, C. Clamer, Y. Dagan, J. Woodhead y O. Zimhoni, sus excavaciones se concentraron principalmente en áreas de los alrededores abiertas por Starkey. Aunque las excavaciones finalizaron en 1987, el trabajo sobre el terreno continuaba con la renovación y reconstrucción de las puertas de la ciudad del período del reino de Judá. ${ }^{17}$

Aunque Ushishkin reconoce que su trabajo fue de menor envergadura que el trabajo de Stankey, ${ }^{18}$ se debe a su labor el establecimiento de una estratigrafía mucho más precisa en lo que respecta a Laquish después del primer milenio.

A estas alturas vale precisar que las excavaciones del montículo se abordaron por áreas identificadas con letras:

Área S: la cual fue la clave para determinar la estratigrafía del montículo. Éste es un largo y angosto sector en el lado accidental del montículo y fue excavado para el examen de los estratos establecidos debajo de la roca (LB).

Área P: incluye el palacio-fortaleza y las estructuras monumentales subyacentes de la edad de Bronce.

Área D: bordea el lado sur-este del palaciofortaleza.

${ }^{16}$ Janet Amitai, ed., Biblical Archaeology Today (Jerusalem: Israel Exploration Society, 1985), 522.

${ }^{17}$ Ushishkin, "Lachish", NEAEHL, 898.

${ }^{18}$ David Ushishkin, "Reassessment of the Stratigraphy and Chronology of Archaeological Sites in Judah in the Light of Lachish III", en Janet Amitai, ed., Biblical Archaeology Today (Jerusalem: Israel Exploration Society, 1985), 143. 
El área G: que contiene las puertas de la ciudad.

El área R: una sección en el centro sur-oeste del montículo, donde el ejército asirio irrumpió a través de las murallas de la ciudad en el 701 AC. ${ }^{19}$

\section{Períodos previos a Hierro II}

Aunque se tratan de períodos que en este estudio no son de nuestro interés, revisaremos muy brevemente lo que se ha dicho de estos períodos con el fin de obtener un mayor contexto de los períodos precedentes.

En las excavaciones de la expedición británica se encontraron tumbas de piedra del período calcolitico y EB I; Ushishkin reporta que fueron encontrados cerca de Laquish herramientas prehistóricas en piedra, además de indicaciones de cerámica neolítica en lugares cercanos a la zona. Cerámica calcolítica encontrada en el montículo indica qué pudo haber ocurrido en el establecimiento en esta época. ${ }^{20}$ Se puede asumir que durante EB II-III el montículo fue extensamente poblado pero de esto muy poco es conocido al presente, señala Ushishkin en otra fuente. ${ }^{21}$

Durante el período EB IV (o MB I período intermedio entre EB - MB) aparentemente el sitio fue abandonado. ${ }^{22}$ Igual suerte se corre con el siguiente período MB específicamente el MB I.

\footnotetext{
${ }^{19}$ Ushishkin, “Lachish”, NEAEHL, 898.

${ }^{20}$ Ibíd.
}

${ }^{21}$ Idem, "Lachish," en David Noel Freedman, The Anchor Bible Dictionary, ed., 6 vols (New York: Doubleday, 1992), 4:115. En adelante ABD.

${ }^{22}$ Ibíd., 4:117. 
Es a partir del MB II - III que el asentamiento al parecer empieza a poblarse nuevamente, hallazgos que datan de MB IIB-C incluyen fortificaciones, un palacio y restos de ocupaciones del borde del montículo. ${ }^{23}$

Las excavaciones revelan una sucesión de tres construcciones superpuestas de templos en los pies del montículo fuera de las defensas en el MB. ${ }^{24}$ Asimismo empieza el afán por la fortificación de la ciudad. ${ }^{25}$ Esto fue colocado en el nivel VIII de la escala de Starkey (ver cuadro en la estratigrafía de Laquish).

Según Finkelstein la reurbanización del MB debe ser entendida como un desarrollo local de cambios socioeconómicos de una mixta sociedad rural-pastoril para luego regresar a una sociedad urbana. ${ }^{26}$

En cuanto al LB parece que Laquish no estaba fortificada en esta época; algunos piensan que había caído en desuso. Aunque también es posible que las casas unidas en forma continua hayan servido como una línea fortificada alrededor de la ciudad. El área excavada en $S$ muestra que era un área abierta en el siglo XIV AC. ${ }^{27}$

${ }^{23}$ Ushishkin, “Lachish”, NEAEHL, 898.

${ }^{24}$ Kathleen M. Kenyon, The Bible and Recent Archaeology (Atlanta, Georgia: Jhon Knox Press, 1978), 25.

${ }^{25}$ Yohanan Aharoni, The Archaeology of the Land of Israel (Philadelphia: The Westminster Press, 1978), 102.

${ }^{26}$ Israel Finkelstein, "The Emergence of Israel: A Phase in the Cyclic History of Canaan in the Third and Second Millennia BCE", en Israel Finkelstein y Nadav Na'aman, eds., From Nomadism to Monarchy: Archaeological and Historical Aspect of Early Israel (Jerusalem: Yad Izhak BenZvi, 1994), 153 
Laquish alcanzó su zenit hacia el fin de la edad LB; pues parece haber sido la más grande ciudad en Canaán después de Hazor que fue destruida en el siglo XIII AC. ${ }^{28}$

Laquish es por primera vez mencionada en una fuente histórica en el papiro Hermitage 1116A, fechado al año 19 ó 20 del faraón Amenhotep II (1427-1404 AC). El mismo menciona ofrendas dadas por los oficiales egipcios a emisarios de diferente ciudades cananeas, entre ellas, Laquish. Varias tabletas cuneiformes contienen cartas enviadas de los reyes de Laquish al Faraón Amnhotep III y a su hijo Amanhotep IV en el XIV siglo AC. Fueron encontradas en los archivos de Amarna en Egipto (cartas 328-332). Otras cartas descubiertas en Tell el-Hesi aparecen siendo enviadas por un oficial egipcio apostado en Laquish. Los nombres de tres reyes de Laquish son mencionados en estas cartas: Zimredah, Shipti-Ba'al, y Yabni-ilu. Aunque la información provista en las cartas es escasa, Laquish parece haber sido uno de los más importantes centros en el sur de Canaán. ${ }^{29}$

El nivel VI fue totalmente destruido con fuego y los habitantes fueron asesinados o deportados. La ciudad fue abandonada y no se restableció más, sino hasta el siglo X AC. ${ }^{30}$

\section{${ }^{28}$ Ibíd. \\ ${ }^{29}$ Ibíd.}

${ }^{30}$ Ushishkin David, "Lachish", 904; Nadav Na'aman, "The 'Conquest of Canaan' in the Book of Joshua and in History", From Nomadism to Monarchy: Archaeological and Historical Aspect of Early Israel, 223; Israel Finkelstein, "State Formation in Israel and Judá", Near Eastern Archaeology 62:1 (1999) 44; Israel Finkelstein, "The Emergence of Israel: A Phase in the Yele History of Canaan in the Third and Second 
La historia de Hierro I es, en parte, la historia bíblica de la transición de las ciudades estado de Canaán a la monarquía unida de Israel. Un principal elemento en su transición fue la fundación de centenares de villas pequeñas. Al parecer en esta época la gente del sur del Levante empezó a desarrollar su propia identidad. ${ }^{31}$

De esta época, son los libros de Josué y Jueces, los que nos cuentan acerca de Laquish relacionándola con los éxitos de Josué. En su primera campaña después de la victoria milagrosa ante Jericó, toma Hai y Gibeón, invade finalmente las montañas de Jerusalem;; ${ }^{32}$ en la referencia a su segunda campaña se precisa que conquistó Libna, Laquish, Eglon, Hebrón y Debir. ${ }^{33}$

\section{Laquish y el período de Hierro II}

Aunque algunos difieren en la terminología de la época ${ }^{34}$ la mayoría de los especialistas concuerdan en

Millennia BCE," From Nomadism to Monarchy: Archaeological and Historical Aspect of Early Israel, 150.

${ }^{31}$ Elizabeth Bloch-Smith y Beth Alpert Nakhai "A Landscape Comes to Life," Near Eastern Archaeology 62:2 (1999) 62.

${ }^{32}$ Jos 1:1-10:28.

${ }^{33}$ Jos 10:29-43.

${ }^{34}$ Israel Finkelstein denomina a este período "Períodos Nacionales Iy II", "Toward a New Periodization and Nomenclature of the Archaeology of the Southem Levant," en J. S. Cooper y G. M. Schwartz, eds., The Study of the Ancient Near East in the Twenty-First Century. The William Foxwell Albright Centenial Conference (Winona Lake, Indiana: Eisenbrauns, 1996), 123; en tanto que Aharoni lo denomina período Israelita, The Archaeology of the Land of Israel, XIX. 
que este período comienza en el año 1000 AC hasta la caída del reino de Judá en 587/6 AC en manos de los babilonios.

\section{Hierro II A}

Comenzaremos este análisis revisando el período Hierro IIA (1000 AC - 925 AC), que según la estratigrafía de Strakey, mejor elaborada por Ushishkin, correspondería al nivel V. ${ }^{35}$ El contexto de esta época lo encontramos en la transición del sistema tribal y de liderazgo de la época de los Jueces hacia una forma de gobierno centralista, teniendo en Saúl su primer rey a finales del siglo $\mathrm{XI} .^{36}$

Las fortificaciones de este período son mayormente del tipo de murallas casemate; hay espacios entre la muralla externa e interna que eran usados como almacenes. ${ }^{37}$ Aunque los asentamientos no son tan marcados en la región, Laquish llegó a ser una de las ciudades más importantes de Judá en los siguientes siglos ${ }^{38}$ a partir de esta época. Respecto a este período Ushishkin reporta lo siguiente:

Los edificios de este nivel fueron mostrados en varias partes del montículo. El establecimiento no fue fortificado, aunque estructuras descubiertas en el área $S$

${ }^{35}$ Ver el cuadro de la estratigrafía propuesto por Ushishkin en el material suplementario.

${ }^{36}$ A. Mazar, Archaeology of the Land of the Bible: 10000-586 B. C. E. (New York: Doubleday, 1992), 368.

${ }^{37}$ Ibíd.

${ }^{38}$ Mazar, Archaeology of the Land of the Bible, 387, 388. 
indican la posibilidad que las casas construidas en el borde del montículo fueron juntadas en una línea defensiva alrededor del sitio. Cerca del santuario solar, Aharoni descubrió un cuarto de culto con un altar de piedra, 4 incensarios levantados y varios cáliz de arcilla; él interpretó los elementos excavados en el área abierta enfrente del cuarto como cúltico. El cuarto de culto fue destruido por fuego, y una evidencia de la destrucción del nivel $\mathrm{V}$ por fuego fue también encontrada en el área S. Sin embargo, los datos arqueológicos son insuficientes para determinar el período de la construcción y la destrucción del nivel V. ${ }^{39}$

Se infiere que este período llegó a su final el año 925 AC con la campaña de Sisac. ${ }^{40}$ Abordaremos los períodos motivo de nuestro mayor interés.

\section{Hierro II B}

Este período abarca aproximadamente desde el 925 AC hasta el 701 AC con la toma de la ciudad a manos de los asirios. Aunque los datos son insuficientes para datar con precisión dos niveles en éste período, se categoriza el nivel IV y III en la época que nos ocupa.

En el nivel IV, Laquish se destaca por la construcción de una gran ciudad fortificada que la constituye en la segunda ciudad en importancia después de Jerusalén. Ushishkin asume que ese cambio se debió a las nuevas consideraciones estratégicas que se levantaron por la di-

${ }^{39}$ Ushishkin, “Lachish”, NEAEHL, 905

${ }^{40}$ Finkelstein, "State Formation in Israel and Judah", 39; Ushishkin, "Lachish", NEAEHL, 905; Mazar, Archaeology of the Land of the Bible, 397. 
visión del reino. ${ }^{41}$ También el registro bíblico da evidencia de ello, cuando señala que Laquish fue una de las ciudades fortificadas por Roboam. ${ }^{42}$

Es notorio, asimismo, en Laquish un gran palacio (B) con un patio exterior que domina el centro de la ciudad; al parecer era la residencia de un gobernador a cargo de una administración provincial. ${ }^{43}$ La construcción de ese palacio fue hecha sobre una base ya levantada (palacio A) llamada estrado por la expedición británica. ${ }^{44}$ Este palacio tenía dos construcciones anexas, una en el norte que era probablemente un almacén y otra en el sur la cual era o un almacén o un establo. ${ }^{45}$

Respecto a las puertas en este estrato, las ciudades usualmente tenían una sola puerta conteniendo casas de 4 a 6 habitaciones hacia adentro; ${ }^{46}$ la puerta de la ciudad de Laquish construida en el lado sur oeste del montículo es la más grande del período de la monarquía

\footnotetext{
${ }^{41}$ Ushishkin, "Lachish”, NEAEHL, 905.

422 Cró 1:5-12, 23.
}

${ }^{43}$ Volkmar Fritz, An Introduction to Biblical Archaeology (Sheffield: JSOT Press, 1996), 150; En cuanto al uso podría ser un motivo religioso, aunque otros piensan que servían como centros administrativos para recaudar los impuestos de la población local, pues "eso es aparentemente lo que muestra Tel Sera y Laquish, al parecer estos dos pueblos dependían directamente de una administración egipcia"; Itamar Singer, "Egyptians, Canaanites, and Philistines in the Period of the Emergence of Israel", en From Nomadism to Monarchy: Archaeological and Historical Aspect of Early Israel, 290. Esto es posible si consideramos la denominación de Sisac al comienzo de este período.

\footnotetext{
${ }^{44}$ Ushishkin, "Lachish", NEAEHL, 906.

${ }^{45}$ Idem, "Lachish", ABD, 4:121.

${ }^{46}$ Fritz, An Introduction to Biblical Archaeology, 150.
} 
encontrada en Israel; ${ }^{47}$ las excavaciones de la puerta en este nivel contenían fragmentos de una bisagra de bronce pertenecientes a una de las puertas de la entrada, y piezas de madera carbonizada, al parecer de acacia - la cual no crece el Laquish. ${ }^{48}$

La ciudad fue rodeada por un sistema de fuertes fortificaciones. La muralla principal (6 mts de espesor) fue construida de ladrillo de barro sobre fundaciones de piedra, también un pozo de alrededor de $44 \mathrm{mts}$ de profundidad fue descubierto en la esquina noreste, junto al revestimiento externo de la muralla, al parecer era el principal suministro de agua para la ciudad. ${ }^{49}$

También a este período se aproximan las vasijas u objetos con motivos humanos que fueron encontrados en Laquish y En-Gadi en el sur, pero que mayormente fueron encontrados en el norte, por lo tanto, algunos creen que se trataría de algún motivo de influencia foranea. ${ }^{50}$

El fin de la ciudad en este nivel, llegó especialmente para las estructuras monumentales; eso se observa en una casa excavada en el área $S$, parece que la destrucción los sorprendió de repente; no obstante no hay evidencia de una deliberada destrucción en este nivel.

${ }^{47}$ Ushishkin, “Lachish”, NEAEHLL, 906.

${ }^{48}$ Ibíd.

${ }^{49}$ Ibíd.

${ }^{50}$ T. A. Holland. "A Study of Palestinian Iron Age Baked Clay Figurines, with Special Reference to Jerusalem: Cave I", Levant (1977), 131. También se encontraron en Beth Shan, Gedi, 'Ein, Hawan, Meguido y Ta'annek'. 
M. Kochavi propuso que la destrucción fue causada por un terremoto similar a lo que ocurrió en el tiempo de Uzías, alrededor del 760 AC. ${ }^{51}$

En los albores del nivel III, la puerta, la fortaleza palacio y la muralla fueron reconstruidos, usando mayormente los fundamentos del nivel IV. Las muchas casas en el área entre el palacio-fortaleza y las puertas de la ciudad reflejan el crecimiento de la población ${ }^{52}$ en este nivel.

La más prominente estructura en la ciudad era la fortaleza-palacio, el estrado A y B que había servido como fundamento para el palacio B ahora era ampliado por la adición del estrado C. Los tres llegaron a ser el fundamento del nuevo palacio, el C. (35 por $76 \mathrm{mt}$.). El almacén del gobernador fue reconstruido y alargado en este nivel. ${ }^{53}$ Shea advierte que Laquish en esta época era una especie de ciudadela real muy similar a Megido y Samaria en el norte de Israel. ${ }^{54}$

En este nivel se encontraron jarras de todo tipo, pero sobresalen las jarras $l m l k,{ }^{55}$ "pertenecientes al rey". Estos estampados eran hechos posiblemente en el mismo lugar donde se hacían las jarras. Casi 30 jarras

${ }^{51}$ Ushishkin, "Lachish" NEAEHLL, 907. Yohanan Aharoni separa este período en el año $800 \mathrm{AC}, \mathrm{IXX}$.

${ }^{52}$ Ibíd, 907.

${ }^{53}$ Ibíd.

${ }^{54}$ William H. Shea, "Sennacherib's Description of Lachish and of Its Conquest," Andrews University Seminary Studies 26:2 (1988) 175. 143.

${ }^{55}$ Ushishkin, "Reassessment of the Stratigraphy and Chronology", 
fueron encontradas en un almacén tanto en Laquish (estrato III) como en Tel Batash. Pero no todas las jarras estaban selladas. ${ }^{56}$

El final de este nivel, se halló por un tiempo en un gran debate. Starkey junto con Inge, Albright y Wright señalaban que aquello se habría llevado a cabo en el año 597 AC en uno de los primeros ataques de Nabucodonosor; ${ }^{57}$ sin embargo Tufnell databa la destrucción en $700 \mathrm{AC}$ fecha que puede ser conectada con la campaña de Senaquerib relatada en 2 Rey 18:14-15. Recientes excavaciones realizadas por la Universidad de Tel Aviv demuestran que Tufnell tenía razón. ${ }^{58}$

Hoy hay consenso entre los eruditos que los hallazgos de destrucción del nivel III corresponden a la destrucción por ejército asirio de Senaquerib alrededor del año $701 \mathrm{AC}^{59}$ ya que al parecer el objetivo senaqueribiano no fue Jerusalén, ${ }^{60}$ en primer lugar sino Laquish estableciendo su campamento en un territorio próximo a la ciudad desde donde era dirigido el ataque. ${ }^{61}$

${ }^{56}$ Mazar, Archaeology of the Land of the Bible, 456.

${ }^{57}$ Ibíd.

${ }^{58}$ Davies, "British Achaeologists", 46.

${ }^{59}$ Lawrence T. Geraty, " Archaeological and the Bible at Hezekiah's Lachish," Andrews University Seminary Studies 25:1 (1987) 35.

${ }^{601}$ Cró 32:9.

${ }^{61}$ David Ushishkin, "The Assyrian Attack on Lachish: The Archaeological Evidence from the Southwest Corner of the Site", Tel Aviv 17 (1990) 54. 
Respecto a esta conquista se precisa que Laquish es el único lugar donde una rampa de sitio asirio fue descubierta. La rampa habría sido construida por mercenarios especializados en estos menesteres. ${ }^{62}$ Tenían un volumen entre 6500 y $9500 \mathrm{~m}^{3}$, invirtiéndose entre 13000 a 19000 toneladas de material para su construcción. ${ }^{63}$ La ubicación fue ideal en términos de topografía, la rampa fue puesta contra la esquina de las murallas de la ciudad, donde los defensores fueron dejados solos en una corta línea de defensa, entonces podrían explotar una pequeña parte de su poder. Senaquerib erigió un campamento que sirvió como su cuartel. Los defensores trataron de detener el trabajo de la rampa arrojando flechas y piedras desde las murallas de la ciudad, más aún ellos intentaron contrarrestar los efectos de la rampa construyendo una contrarampa, levantándola inclusive más alto que las murallas de la ciudad. ${ }^{64}$

Al final Laquish cayó luchando, pero los asirios tuvieron también pérdidas considerables. ${ }^{65} \mathrm{El}$ incendio de la ciudad es evidente en todas las estructuras del nivel III; grandes cantidades de vasijas fueron dejadas en las ruinas ${ }^{66}$ precisa Ushishkin.

${ }^{62}$ Ephraim Stern, Archaeology of the Land of the Bible: The Assyrian, Babylonian, and Persian Periods (732-333 B.C.E.) Vol II, The Anchor Bible Reference Library, ed. David Noel Freedman (New York: Doubleday, 2001), 5.

${ }^{63}$ Ushishkin, “The Assyrian Attack on Lachish”, 64.

${ }^{64}$ Amihai Mazar. "The fortification of Cities in the Ancient Near East", en Jack Sasson, ed., Civilizations of the Ancient Near East, 2 vols. (Massachusetts: Hendrickson Publishers, 1995), 2:1535.

${ }^{65}$ Chaim Herzog y Mordechai Gichon, Battles of the Bible (New York: Random House, 1978), 142.

${ }^{66}$ Ushishkin, "Lachish", NEAEHL, 907. 
Su falla para conquistar Jerusalem probablemente determinó la conmemoración de la conquista de Laquish en un enorme relieve, localizado en el cuarto central del palacio de Senaquerib en Nínive; ${ }^{67}$ en éste las tropas asirias son mostradas avanzando de la izquierda, la ciudad es atacada sobre ruedas y se observa a Senaquerib enfrente de su campamento recibiendo la ciudad. ${ }^{68}$

\section{Hierro II C}

Después del final del estrato III Laquish fue abandonada. Es probable que el establecimiento haya sido renovado y fortificado durante los días de Manasés o posiblemente en los días del rey Josías (639-609 AC); ${ }^{69}$ el estrato de la ciudad fue más pobre, menos poblado y más pequeño.

Al parecer la ciudad fue reconstruida sobre las ruinas de las puertas de los niveles IV y $\mathrm{II}^{70}$ el revestimiento exterior de la muralla fue reparado y una nueva muralla fue reconstruida en el borde del montículo encima de las ruinas de la muralla de los niveles IV y III. El palacio fortaleza no fue renovado, permaneció apilado en ruinas en el centro de la ciudad..$^{11}$

${ }^{67}$ Mazar, "The Fortification of Cities in the Ancient Near", 2:1535.

${ }^{68}$ T. R. Mitchell, Biblical Archaeology: Documents from the British Museum (New York: Cambriedge University Press, 1998), 59.

${ }^{69}$ Aharoni, The Archaeology of the Land of Israel, 272.

${ }^{70}$ Ushishkin, "Lachish", 909.

${ }^{7}$ I bid. 
Para este tiempo el imperio asirio que había sido el agente destructor de Laquish según lo visto en el nivel anterior, había sido sucedido por el ambicioso imperio Neo-babilónico y las ciudades palestinas pronto volvieron a ser asechadas por las fuerzas destructoras. ${ }^{72}$

Así como otras ciudades amuralladas que pronto cayeron ante el poder babilónico, Laquish sucumbió ante el fuego enemigo, ${ }^{73}$ siendo una vez más destruida la restaurada ciudad. ${ }^{74}$ Las excavaciones mostraron dentro de la ciudad, almacenes y casa habitaciones. ${ }^{75}$ También se encontraron numerosas vasijas quemadas bajo la ruina de las casas; entre la cerámica es notable el almacén de jarras con impresiones con flores. ${ }^{76}$

En un cuarto de guardia, entre la puerta exterior e interior de la ciudad, en la capa de cenizas se encontró una colección de ostraca o fragmentos de vasijas utilizadas para escribir mensajes; se encontró un total de 18 ostraca $^{77}$ comúnmente conocidas como las cartas

\section{${ }^{72}$ Ver Jer 34:7}

${ }^{73}$ Leona Glidden y David Noel Freedman Running, eds., William Foxwell Albright: A Twentieth-Century Genius (New York: Morgan Press, 1975), 194. Albright precisó que Laquish fue la última en caer antes que los babilonios alcanzaran Jerusalén; aunque él se equivocó al señalar que el fin del nivel dos correspondía al ataque del mismo imperio en el año 597 AC.

${ }^{74}$ Se puede inferir que el asecho babilónico fue similar al asirio en cuanto a la construcción de una rampa para tomar la ciudad. Gösta W. Ahlströn y Diana Edelman, eds., The History of Ancient Palestine (Minneapolis: Fortress Press, 1993), 795.

${ }^{75}$ Mazar, Archaeology of the Land of the Bible, 434.

${ }^{76}$ Ushishkin, "Lachish", NEAEHL, 909.

${ }^{77}$ Kathleen M. Kenyon, Arqueología en Tierra Santa (Barcelona: Ediciones Garriga, 1963), 295. 
de Laquish. Se presume que estos documentos fueron escritos rápidamente antes que Laquish fuera capturada y destruida por los babilonios en 578/6 AC. La mayoría son reportes enviados a un comandante judío en Laquish (Yaosh/Yaush) por uno de sus subordinados que estaba en otra posición. ${ }^{78}$ El mensaje reporta que las señales desde Laquish están siendo advertidas, pero aquellas desde Aseka, ${ }^{79} 15 \mathrm{kms}$ al nor-este de Laquish no pueden ser vistas, ${ }^{80} \sin$ duda esto refleja la crítica situación de esta parte del territorio judío en esta época.

Después de su destrucción a manos de los caldeos, fin de lo que se conoce como nivel II, parece que el sitio fue abandonado por algún tiempo; ${ }^{81}$ volviendo a la palestra en el período persa donde se establecieron un grupo de exiliados que retornaron de Babilonia, ${ }^{82}$ lo que Tufnull reconoce como nivel I. ${ }^{83}$

\section{Cultura material de Laquish}

Es el objetivo de toda misión arqueológica al excavar un determinado lugar encontrar restos materiales que pueden brindar una evidencia "tangible" de tal ocual lugar o

${ }^{78}$ Gerald L. Mattingly, "Lachish," Diccionary of the Ancient Near East (Philadelphia, Pennsylvania: University of Pennsylvania Press, 2000), 173.

${ }^{79}$ Tell ez-Zakariyeh. Rainey, "Historical Geography", 360.

${ }^{80}$ Ahlström, The History of Ancient Palestine, 796.

${ }^{81}$ Ushishkin, "Lachish", 910.

${ }^{82} \mathrm{Neh} 11: 30$.

${ }^{83}$ Stern, Archaeology of the Land of the Bible, 447. 
época según el interés de los exploradores. Los excavadores en Laquish, en sus diferentes proyectos, no fueron privados de esta tremenda satisfacción; en las postrimerías de este estudio, este investigador puede dar fe de la riqueza de este yacimiento en cuanto a cultura material.

\section{La cerámica del Nivel III}

Es interesante notar que aunque el reino del norte era más rico y más extenso, Judá lo excedió en grande proporción a su cultura material. ${ }^{84}$ Laquish en particular fue uno de los lugares donde se encontró grandes proporciones de cerámica; en el nivel III se encontraron jarras de todo tipo, sobresaliendo las jarras $l m l k,{ }^{85}$ que algunos piensan que tuvieron influencia de sus pares del norte o de los fenicios.

Las jarras fueron probablemente producidas en los años precedentes a la invasión asiria, y su función según algunos investigadores fue la de contener provisiones reales, particularmente vinoy/o provisiones para el ejército de Ezequías asentado donde se pensaba que el ataque de Senaquerib sería probable. ${ }^{86}$ Según las descripciones que ofrece Mazar las jarras tenían un cuello angosto, amplios hombros, una base angosta y 4 agarraderas y su capacidad variaba entre 12 y 14 galones ( 45 y 53 lts). ${ }^{87}$

${ }^{84}$ Aharoni, The Archaeology of the Land of Israel, 241.

${ }^{85}$ Ushishkin, "Reassessment of the Stratigraphy and Chronology of", 143; El autor piensa que las jarras no fueron producidas directamente en Laquish sino en un lugar próximo a éste." Lachish" NEAEHL, 909; Otros ven en su arte cierta influencia de sus pares del norte o de los fenicios, Holland, "A Study of Palestinian Iron Age Baked Clay", 131.

${ }^{86}$ Mazar, Archaeology of the Land of the Bible, 457. 
Pero lo que más resalta de estas jarras son sus impresiones "para el rey" o "pertenecientes al rey", lmlk. Centenares de estos estampados son conocidos; ellos fueron impresos en una forma un tanto descuidada en las asas de las jarras en una típica forma vertical. ${ }^{88}$

Los sellos con los que se hacían estos estampados, que también se han encontrado en las excavaciones, ${ }^{89}$ tenía principalmente dos motivos: uno tiene una forma de escarabajo y otro parecía un disco solar. La palabra lmlk era puesta sobre el símbolo y debajo se escribía uno de los 4 nombres de los siguientes lugares: Hebrón, Ziph, Socchoh y mmts. ${ }^{90}$

Aunque en menor proporción, se encontraron también cerámicas estampadas con nombres personales. Fueron notados en las excavaciones diferentes nombres, cuyos dueños pueden haber sido quienes estaban involucrados directamente en la manufactura de jarras, ${ }^{91}$ en total 413 estampados $l m l k$ y 65 estampados "privados" fueron encontrados en Laquish. ${ }^{92}$

${ }^{87}$ Mazar, Archaeology of the Land of the Bible, 456. Casi 30 jarras fueron encontradas tanto en Laquish, en el estrato III, como en Tel Batash.

${ }^{88}$ Mazar, Archaeology of the Land of the Bible, 456.

${ }^{89}$ Ushishkin señala que A. Lemaire ha mostrado recientemente 22 diferentes estampas que fueron usados para hacer impresiones $l m l k h$. "Lachish", 909.

${ }^{90}$ Mazar, Archaeology of the Land of the Bible, 456. El autor precisa que las dos primeras ciudades son conocidas localizadas en las montañas de Hebrón. Sochoh probablemente se ubicaría en la Sefela y las últimas es desconocida, aunque quizá haya sido un título de Jerusalem algo como "gobernación".

${ }^{91}$ Mazar, Archaeology of the Land of the Bible, 457.

${ }^{92}$ Ushishkin, “Lachish" NEAEHL, 909. 
Ushishkin recuerda que a la luz de estos hallazgos los excavadores presumen las siguientes conclusiones: (a) todas las jarras fueron usadas corrientemente; (b) fueron producidas durante el reinado de Ezequías, poco antes del 701 AC porque ellas fueron encontradas bajo el nivel III de destrucción; (c) la capacidad de jarras estampadas no es consistente; varía entre 39.75 y 51.8 lts; (d) siendo que las estampas lmlk y las estampas "privadas " fueron impresas en jarras semejantes, los dueños de los sellos privados pueden haber sido oficiales en el centro de producción o los alfareros, y (e) muchos de los sellos lmlk y privados fueron descuidadamente estampados, indicando que no hubo una necesidad especial de ser leídos. ${ }^{93}$

\section{Las inscripciones del nivel II}

Razón tiene Stern cuando señala que de los hallazgos epigráficos del siglo VI uno de sus mejores archivos viene de Laquish, ${ }^{94}$ sus cartas son usadas como ejemplos de inscripciones datadas del año 587 AC guardando cierta semejanza con inscripciones de Mesa y Siloam. ${ }^{95}$

En enero de 1935 los excavadores encontraron 18 ostracas que eran cartas o listas de nombres en escritura hebrea antigua del tiempo de Jeremías. Tres ostracas

\section{${ }^{93}$ Ibíd.}

${ }^{94}$ Mazar, Archaeology of the Land of the Bible, 170.

${ }^{95}$ Joel F. Drinkard, Jr., "Epigraphy as a Dating Method," en Joel F. Drinkard Jr., et al., eds., Benchmarks in Time and Culture (Atlanta, Georgia: Scholars Press, 1988), 425. 
más fueron encontradas en $1937-38,{ }^{96}$ siendo publicadas con admirable celeridad. Su contenido ha contribuido al conocimiento de las costumbres de la sociedad israelita así como al conocimiento del desarrollo de la ortografía hebrea. ${ }^{97}$

N. H. Torczyner, Lemaire y otros creen que estas cartas fueron enviadas "a mi señor Yaush" un comandante militar en Laquish poco tiempo antes de la conquista babilónica por un subordinado establecido en algún punto donde el podía ver señales de Laquish y Azeca. Sin embrago, Yadin arguye que las cartas de Laquish fueron copias o borradores de cartas enviadas desde Laquish a un comandante en Jerusalén. ${ }^{98} \mathrm{Al}$ evaluar La ostraca IV que literalmente dice: "y permita ( $\mathrm{mi}$ señor) conocer que nosotros vigilando las señales de Laquish, de acuerdo a todas las indicaciones que mi señor ha dado, no podemos ver Azeca" ${ }^{99}$ claramente se puede inferirse que Torczyner tenía razón.

Varias otras inscripciones en cerámica fueron descubiertas en almacenes de este nivel II cerca de las puertas de la ciudad. Dos de ellas mencionan fechas: "en el cuarto" y "en el noveno" probablemente para

${ }^{96}$ Davies, "British Archaelogists", 44.

${ }^{97} \mathrm{P}$. Bordreuil, F. Israel y D. Pardee, "King's Command and Window's Plea: Two New Hebrew Ostraca of the Biblical Period", Near Eastern Archaeology 61:1 (1998) 2.

${ }^{98}$ Ushishkin, “Lachish”, 910.

${ }^{99}$ W. F. Albright, trad., "Palestinian Inscription," en James B. Pritchard, ed., Ancient Near Eastern Texts (Princenton, New Jersey: Princenton University Press, 1955), 322. 
referirse a los años de reinado de Zedequías rey de Judá. ${ }^{100}$

Asimismo se ha encontrado un sello con la descripción lgdlyhw 'shr 'l hbyt "Gedalyahu quien está sobre la casa" refiriéndose al individuo que los caldeos pusieron como gobernador sobre Judá después de su destrucción; ${ }^{101}$ el nombre de Jehová es también registrado en ostracas de este siglo. ${ }^{102}$

\section{Arquitectura y otros}

En el corazón de la ciudad de Laquish, desde tiempos anteriores a Hierro II, un gran complejo de edificios fue levantado, el cual proveía una gama de servicios para la corte, llámese: negocios administrativos, guardia local, etc.

La principal estructura era el gran palacio erigido en un alto estrado. Mazar precisa que era el palacio A. La primera fase de su construcción, era una estructura cuadrada de 32×32 asentado en un estrado de piedra. Esto parece haber sido construido en el nivel V. En la siguiente fase de su construcción (Palacio B, nivel IV) parece que fue alargado al sur unos $44 \mathrm{~m}$. En cambio en la tercera fase (Palacio C, Nivel III) fue alargado hacia el este. El resultado fue una dimensión final de 36×76 m la más grande construcción de la edad de Hierro dada a

${ }^{100}$ Ushishkin, “Lachish", 910.

${ }^{101}$ Charles F. Pfeiffer, ed., The Biblical World (Grand Rapids, MI: Baker Book House, 1976), 515. 
conocer en Israel. ${ }^{103}$ Se considera pertinente recapitular esto, puesto que el palacio Israelita descubierto en Laquish es el de mayor extensión hasta ahora descubierto "debido a la falta de datos arquitectónicos concernientes a los palacios y a los edificios oficiales situados en Jerusalén. Este palacio puede servir como modelo para las residencias reales que pueden haber existido en la capital judia"104 por esta época.

Vale la pena señalar algo sobre la cultura "gastronómica" y la buena dieta de los laquianos como Beer-Sheba y Hai entre otros. En Laquish fue encontrado cereal y polen en la edad de Hierro; ${ }^{105}$ además, aparte de la planta de la oliva, muy popular en la zona, también se encontraron semillas de trigo y varias clase de legumbres, tales como: frijol (¿pallares?) y granos de garbanzo. ${ }^{106}$

\section{Conclusión}

Al terminar esta "excavación", este investigador queda convencido que el estudio de la arqueología de Israel es muy importante, ya que gracias a ella no sólo podemos conocer un poco de la historia del país sino

${ }^{103}$ Mazar, Archaeology of the Land of the Bible, 428.

${ }^{104}$ Ronny Reich, "Palaces and Residencies in the Iron Age", en Aharon Kempinski y Ronny Reich, eds., The Architecture of Ancient Israel From the Prehistoric to the Persian Periods (Jerusalem: Israel Exploration Society, 1992), 208.

${ }^{105}$ Avraham Horowitz, "Palynology," en Joel F. Drinkard Jr., et al., Benchamarks in Time and Culture, eds. (Atlanta, Georgia: Sholars Press, 1988), 277.

${ }^{106}$ Zohar Amar, "Agricultural Realia in Light of the Lachish Relief", Ugarit-Forschungen 31 (1999) 2, 3. 
que "de paso", como diría Ushishkin, podemos entender mejor "la Biblia y el mundo de la Biblia". ${ }^{107}$

Bien es sabido que la historicidad de la monarquía del siglo $X$ ha sido puesta en tela de juicio ${ }^{108}$ y últimamente los eventos del pueblo judío en el siglo VII están siendo reducidos a simples relatos ideológicos del siglo IV; frente a esto valdría la pena preguntarse, ¿qué hacer con las evidencias que la arqueología ofrece con respecto a este período Israelita?

Es verdad que es muy escaso el material que data de tiempos monárquicos y particularmente de su capital Jerusalén. Sin embargo, épocas y lugares subsiguientes están tan ampliamente documentados que sería poco prudente ignorar su contribución.

Laquish es uno de esos lugares. Su inscripción in situ que la identifica como tal, las evidencias de sus destrucciones por los poderosos reinos de turno y su abundante cerámica $l m l k$ que no excede la era de predominio babilónico ${ }^{109}$ son pruebas más que evidentes para la historicidad de la época.

${ }^{107}$ Ushishkin, "Reassessment of the Stratigraphy and Chronology of Archaeological Sites in Judah in the of Lachish III", 142.

${ }^{108}$ Finkelstein advierte que minimistas como Jamieson-Drake llegaron a la conclusión de que Judá no pudo haber sido fundada antes del siglo VIII AC, igual posición tiene Devies, Tompson y Lemche. "State Formation in Israel and Judá", 39.

${ }^{109}$ Ya habían quedado fuera de uso en el nivel II. Aharoni, The Archaeology of the Land of Israel, 241. En el apéndice de este trabajo se incluyen muestras de cerámica tomadas de este autor, figura 6. Además se incluye en el apéndice la foto de James Starkey, figura 1, una tableta de la carta de Laquish, figura 2; la reconstrucción de Laquish en el nivel III, figura 3; un plano de la ciudad, figura 4; un plano del templo, figura 5; los 3 últimos tomados de $A B D, 4: 122,116$ y 119. 